\title{
Two Cases of Arnold-Chiari Malformation with Respiratory Failure
}

\author{
Sinem Iliaz ${ }^{a}$, Raim Iliaz ${ }^{b}$ Mustafa Erelel ${ }^{\mathrm{a}}$ \\ a Pulmonary Medicine Department, Istanbul Medical Faculty, Istanbul University, Istanbul, Turkey \\ bInternal Medicine Department, Istanbul Medical Faculty, Istanbul University, Istanbul, Turkey
}

\begin{abstract}
:
Arnold-Chiari malformation is defined as downward displacement of the brainstem and cerebellum through the foramen magnum. It has different clinical presentations and four subtypes. It is known that downward migration of posterior fossa components through the foramen magnum and associated lower cranial nerve palsy and brainstem compression can cause respiratory failure. Acute respiratory failure could mark the onset of the disease. Posterior fossa decompression performed to treat primary disease can improve the central sleep abnormalities. As respiratory failure is rarely seen, this paper presents two cases of Arnold-Chiari malformation with respiratory failure.
\end{abstract}

Keywords: Arnold-Chiari malformation, respiratory failure, hypercapnia

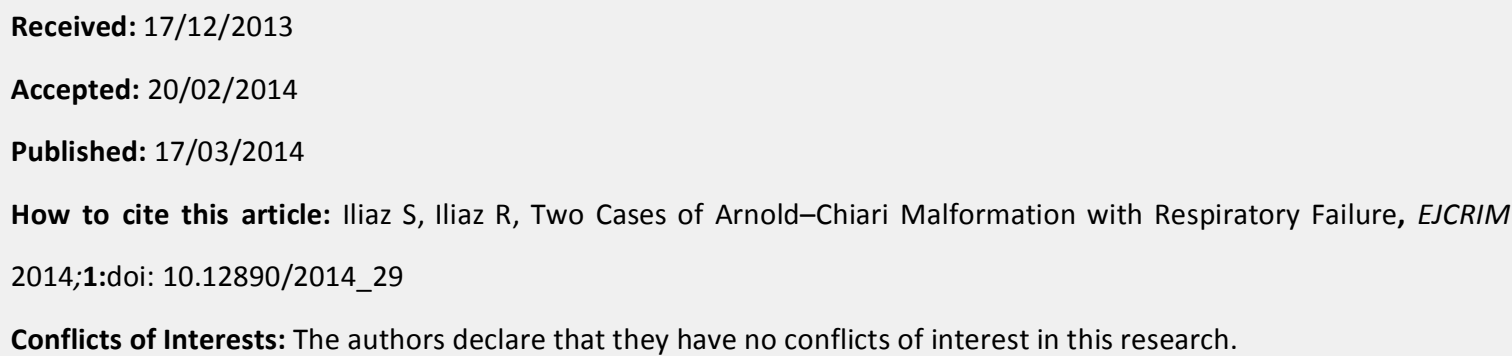




\section{Introduction}

Arnold-Chiari malformation (ACM) is defined as downward displacement of the brainstem and cerebellum through the foramen magnum ${ }^{1}$. Patients are mostly referred to a physician with weakness, loss of sensation, bulbar dysfunction, headache, imbalance, gait disturbance and syncope, but also with acute respiratory failure ${ }^{2}$. Adolescents and adults who have ACM but no symptoms initially may, later in life, develop signs of the disorder. Respiratory failure is rarely seen, but it can be present at onset. Respiratory failure is mostly due to central hypoventilation and apnoea, although an obstructive pattern is occasionally reported ${ }^{3,4}$. It is known that downward migration of posterior fossa components through the foramen magnum and associated lower cranial nerve palsy and brainstem compression can cause respiratory failure ${ }^{3}$. This paper reports two ACM patients with respiratory failure and sleep apnoea syndrome treated surgically.

\section{Case 1}

A 35-year-old man, affected by ACM and syringomyelia, who had been operated on 4 years before, had onset of dyspnoea. Subsequently, diaphragm plication was performed for left diaphragm paralysis. The patient had been on Bilevel Positive Airway Pressure (BIPAP) treatment with pressure of $11 / 5 \mathrm{mmHg}$ at night for his hypercapnic respiratory failure. The patient was

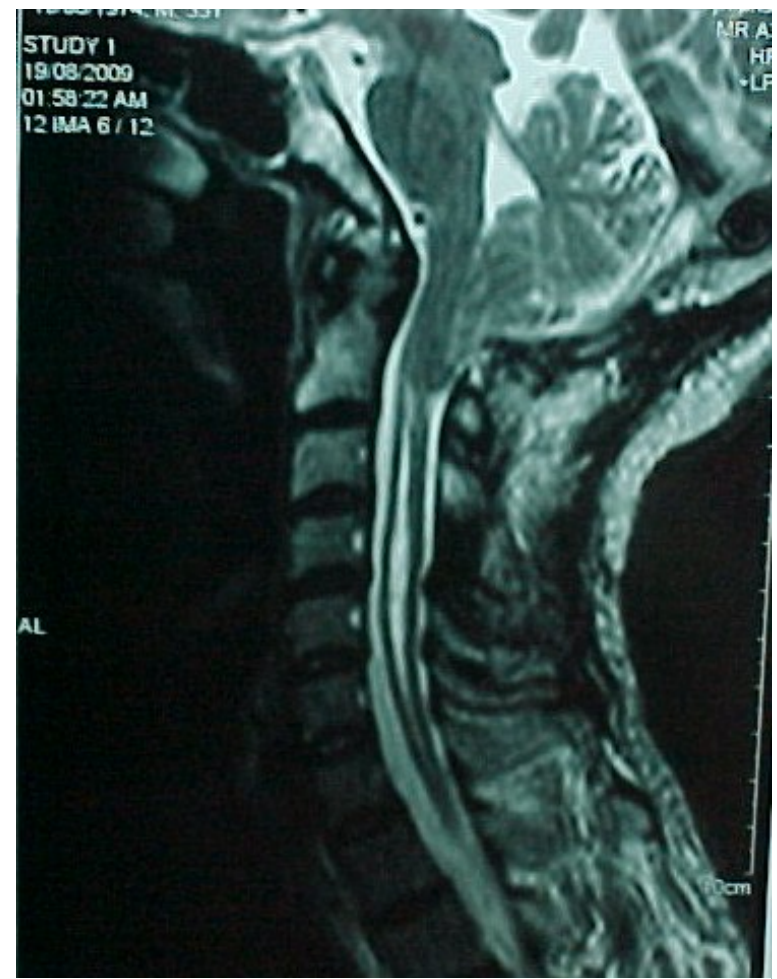

Figure 1: Cranial MRI of the first case showing downward displacement of cerebellar tonsils and syringomyelia. referred to our clinic with dyspnoea, long-lasting purulent sputum and a history of repeated respiratory infections. The physical examination revealed slow motion and slow speech. He was otherwise normal. His arterial blood gas analysis in room air showed $\mathrm{PaO}_{2} 80$ $\mathrm{mmHg}, \mathrm{PaCO}_{2} 52 \mathrm{mmHg}, \mathrm{pH} 7.40$ and $\mathrm{SaO}_{2}$ 95\%. A chest $X$-ray showed left diaphragmatic elevation. His lab values were normal. Forced Vital Capacity (FVC) was $2350 \mathrm{ml}$ (50\%), Forced Expiratory Volume 1 second (FEV1) $2280 \mathrm{ml}$ (60\%) and FEV1/FVC 0.97. The 6-minute walking test did not cause desaturation for a walking distance of $320 \mathrm{~m}$. There was no additional pathology in cranial and whole spinal MRI (Fig. 1). Polysomnography disclosed hypopnoea and apnoea periods of central origin that persisted with BIPAP $11 / 5 \mathrm{mmHg}$. 
The apnoea-hypopnoea index (AHI) was 31.4 (severe sleep apnoea syndrome), well controlled with $16 / 6 \mathrm{mmHg}$ pressure.

\section{Case 2}

A 35-year-old female presented with sleepiness; swelling of legs, face and neck; cyanosis of fingers and lips; and right pleuritic chest pain. A physical examination showed bilateral +/+ pretibial oedema, jugular venous distention and decreased breath sounds bilaterally in the middle-lower pulmonary field. She had an abnormal gait with broad steps.

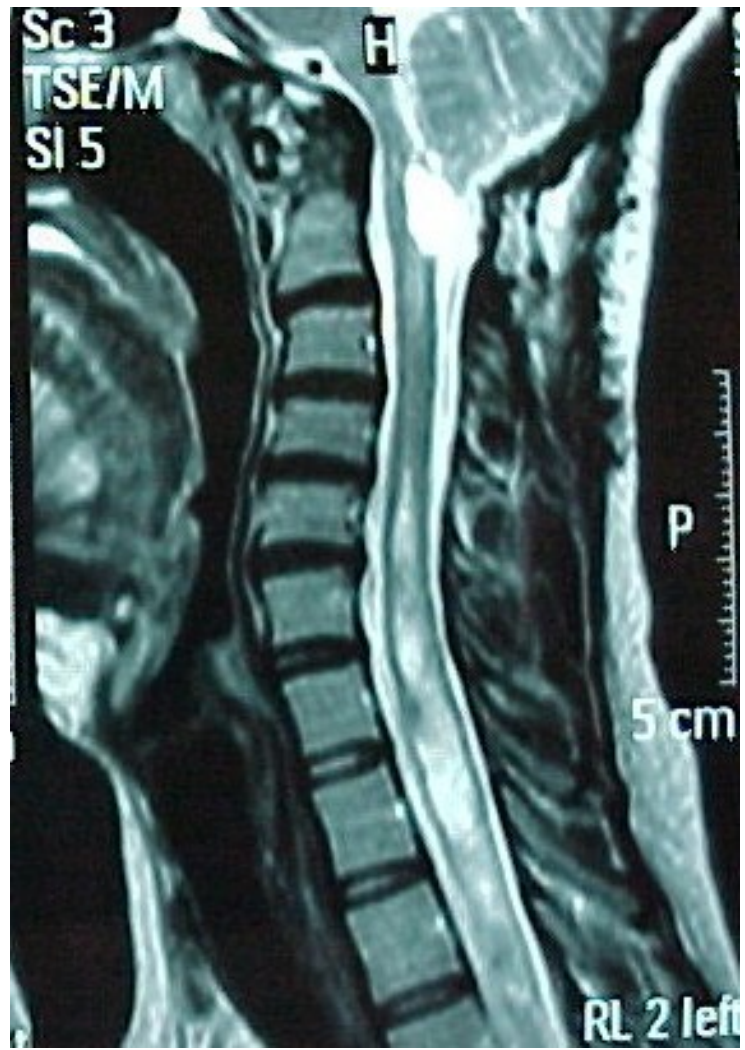

Figure 2: Cranial MRI of the second case showing a cystic

formation near the brainstem and syringomyelia

Her past medical history disclosed posterior fossa decompression surgery for ACM and syringomyelia 9 years before. She was followed for a nonfunctional hypophysial adenoma. Her vital signs were normal. $\mathrm{PaO}_{2}$ was $31 \mathrm{mmHg}$, $\mathrm{PaCO}_{2} 75 \mathrm{mmHg}, \mathrm{pH} 7.32$ and $\mathrm{SO}_{2} 61 \%$. The patient was treated with BIPAP and oxygen for hypercapnic respiratory failure causing hypoxia. The right costophrenic angle was blunted. The thorax CT demonstrated pneumonic infiltration in her middle pulmonary lobe, right pleural effusion and associated compression atelectasis. The lab exams showed C-reactive protein 15 $\mathrm{mg} / \mathrm{dl}$ and no leukocytosis. Moxifloxacin $400 \mathrm{mg}$ i.v.q.d. was started. FVC was $1970 \mathrm{ml}$ (75\%), FEV1 $1340 \mathrm{ml}$ (59\%) and FEV1/FVC 0.68. During the 6-min walking test, there was no desaturation for a walking distance of $280 \mathrm{~m}$. Pulmonary arterial pressure (PAP) ranged between 35 and $45 \mathrm{mmHg}$ and echo examination showed a dilated right atrium. Daytime $\mathrm{SaO}_{2}$ rose to $98 \%$ during Noninvasive mechanical ventilation (NIMV). With BIPAP $14 / 5 \mathrm{mmHg}, \mathrm{PaO}_{2}$ was $62 \mathrm{mmHg}, \mathrm{PaCO}_{2} 74 \mathrm{mmHg}, \mathrm{pH} 7.45$ and $\mathrm{SO}_{2}$ 92\%. The patient was allowed to breathe room air in the daytime, aided with BIPAP $14 / 5 \mathrm{mmHg}$ at night. In her cranial and whole spinal MRI, there was a cystic formation near the brain stem (Fig. 2), which could have caused brain stem compression and central hypoventilation, although brainstem auditory evoked potential (BAEP) was normal. Polysomnography demonstrated severe sleep apnoea (AHI 49.4) and desaturation, which improved with BIPAP $15 / 8 \mathrm{mmHg}$ plus oxygen $2 \mathrm{l} / \mathrm{min}$. 


\section{Discussion}

ACM is the elongation of the brain stem and cerebellum through the foramen magnum ${ }^{1}$, which can damage central respiratory control, leading to respiratory disorders, respiratory failure and death $^{3}$. The associated cervical syringomyelia can also lead to respiratory failure due to muscular weakness and phrenic nerve disease. Posterior fossa decompression performed to treat the primary disease can improve central sleep abnormalities ${ }^{3}$. Levitt and Cohn reported improvement in sleep apnoea after surgical treatment of ACM, but the AHI (14) was less severe than that of our patients ${ }^{5}$. Improvement in both central and obstructive sleep disorders occurs postoperatively, indicating that ACM is responsible for both ${ }^{4}$. However, respiratory failure may not improve as much as sleep disturbance, such that patients may need NIMV and oxygen support. Both of the cases herein described had ongoing respiratory failure after surgery. BIPAP was introduced postoperatively, but higher-pressure NIMV was needed afterwards. The second case had respiratory failure triggered by pulmonary infection, as already reported by Alvarez et al. ${ }^{3}$.

Sleep abnormalities of ACM can be adequately treated with NIMV (CPAP, BIPAP) at night-time, with oxygen supplementation, as suggested by polysomnographic studies. As the symptoms of this disease can be nonspecific, it is important to keep this in mind when dealing with central nervous system symptoms in young adults with hypercapnic respiratory failure. As in our cases, patients operated on for ACM must be evaluated for chronic respiratory failure and sleep apnoea after surgery, as respiratory problems can progress in time, avoiding respiratory arrest. As demonstrated by one of our patients, life-threatening respiratory failure may occur after an infectious trigger in the absence of previous respiratory symptoms.

\section{Learning points}

- Arnold-Chiari malformation can cause central nervous system sleep abnormalities and respiratory failure.

- In young patients with hypercapnic respiratory failure, neurologic causes must be sought.

- In Arnold-Chiari malformation, respiratory problems may diminish or persist postoperatively.

\section{REFERENCES}

1. Chiari H. Ueber veranderungen des kleinhirns infolge von hydrocephalie des grosshirns, Dtsch Med Wochenschr 1891;42:1172-1175.

2. Naidich TP, Zimmerman RA. Common congenital malformations of the brain, in Magnetic Resonance Imaging of the Central Nervous System. New York: M. Brant-Zawadzki and D. Norman, 


\section{European Journal \\ of Case Reports in \\ Internal Medicine}

Raven Press, 1987, pp. 131-150.

3. Alvarez D, Requena I, Arias M, Valdes L, Pereiro I, De la Torre R. Acute respiratory failure as the first sign of Arnold-Chiari malformation associated with syringomyelia, Eur Respir J 1995;8:661663.

4. Gosalakkal JA. Sleep-disordered breathing in Chiari malformation type 1, Pediatr Neurol 2008;39:207-208.

5. Levitt P, Cohn MA. Sleep apnoea and the Chiari I malformation: case report, Neurosurgery 1988;23:508-510. 